\title{
Acoustic radiation force imaging (ARFI) in the non-distended bladder does not predict abnormal urodynamic parameters in children
}

\author{
Courtney S. Streur, $M D, M S^{\prime}$; Ethan A. Smith, $M D^{2}$; Jonathan R. Dillman, $M D, M S c^{2}$; Kate H. Kraft, $M D^{\prime}$
}

'Department of Urology, University of Michigan, Ann Arbor, MI, United States; 2 Department of Radiology, Cincinnati Children's Hospital Medical Center, University of Cincinnati College of Medicine, Cincinnati, $\mathrm{OH}$, United States

Cite as: Streur CS, Smith EA, Dillman JR, et al. Acoustic radiation force imaging (ARFI) in the nondistended bladder does not predict abnormal urodynamic parameters in children. Can Urol Assoc J 2022;16(1):E15-9. http://dx.doi.org/10.5489/cuaj.7218

Published online August 26, 2021

\section{Abstract}

Introduction: Acoustic radiation force imaging (ARFI) is a recently developed form of ultrasound imaging that allows in vivo measurement of tissue stiffness. This technology could be useful at predicting bladder compliance in children. We hypothesize that tissue stiffness, as measured by ARFI, correlates with abnormal bladder compliance and capacity in patients with bladder dysfunction.

Methods: Patients who presented for cystometrography (CMG) underwent ARFI of the bladder wall. Nine bladder wall shear wave speed (SWS) measurements were acquired using point and 2D ultrasound shear wave elastography. The mean for each ARFI technique was correlated to bladder compliance, calculated using Wahl's dimensionless number. ARFI parameters also were correlated with bladder capacity.

Results: A total of 25 patients were enrolled. Mean age at time of enrollment was $4.2 \pm 3.9$ years (range two months to 15 years). There was no significant correlation between bladder compliance and point shear wave speed measurements $(r=-0.22, p=0.31)$ or $2 \mathrm{D}$ shear wave speed measurements $(r=-0.35, p=0.1)$. A total of 19 patients had bladder capacity below expected bladder capacity (EBC). There was no significant correlation between bladder capacity and point shear wave speed measurements $(r=-0.08, p=0.7)$ or $2 \mathrm{D}$ shear wave speed measurements $(r=-0.36, p=0.09)$.

Conclusions: Our results did not demonstrate a significant correlation between bladder wall ARFI shear wave measurements and bladder compliance or bladder capacity. Further studies are warranted to determine whether ARFI may be used to predict abnormal urodynamic parameters in children.

\section{Introduction}

Patients with bladder dysfunction secondary to abnormal innervation or bladder outlet obstruction are at risk of developing renal deterioration from elevated intravesical pressure and poor bladder compliance. ${ }^{1,2}$ In this clinical setting, such patients are followed closely with imaging (typically conventional gray-scale ultrasound and voiding cystourethrography) and urodynamic testing to guide management, with the goal of preventing renal deterioration.

Currently, urodynamic testing serves as the gold standard for functional assessment of the lower urinary tract and is the only available method capable of reliably identifying patients at risk for upper tract deterioration due to poor bladder compliance. Bladder dynamics often change with age and growth, so repeat urodynamic studies are required throughout childhood. Urodynamic testing is especially challenging in pediatric patients, who often have difficulties tolerating the procedure and may require various distraction methods, Child Life Specialist support, or even sedation. Therefore, a non-invasive method to evaluate urodynamics and bladder wall fibrosis in pediatric patients would be ideal.

Acoustic radiation force imaging (ARFI), or ultrasound shear wave elastography, is a type of ultrasound assessment that can provide quantitative measurements of tissue stiffness. This technology evaluates the deformability of tissue by shear waves, and thus, it is affected by both physiological (bladder filling) and pathological (changes in histology) alterations in the bladder. ARFI is currently most widely used to evaluate liver fibrosis and has potential benefits in other organ systems as well. ${ }^{3-5}$ As poor bladder compliance is thought to be due to an increase in fibrosis in the bladder wall, this ARFI could potentially be helpful in the evaluation of a neuropathic bladder. ${ }^{6,7}$ Sturm et al recently published their experience with this technology and found ultrasound shear wave elastography bladder measurements correlate well with bladder storage pressure. ${ }^{8}$ It is possible that ARFI could serve to restrict invasive testing to only those patients shown to be at high risk for deterioration of the upper urinary tract or obviate the need for urodynamic testing altogether in some patients.

The aim of this study is to compare bladder wall stiffness, as measured by ARFI, with parameters obtained at urodynamic testing. We hypothesize that increased bladder wall shear wave speed measurements (e.g., increased tissue stiff- 
ness), as measured in the non-distended bladder, correlate with decreased estimated bladder capacity and decreased bladder compliance.

\section{Methods}

\section{Subjects}

Following institutional review board approval, subjects were enrolled prospectively in this feasibility study between January 2013 and September 2016. Patients were eligible for enrollment if presenting to the pediatric urology clinic for routine filling cystometrography (CMG) as part of their evaluation for bladder dysfunction and families consented to undergoing ARFI. Informed consent for ARFI ultrasound was obtained from the parents. When applicable, informed assent was also obtained from the patients. Patients were excluded if there was any history of prior reconstructive bladder surgery (e.g., bladder augmentation, ureteral reimplantation).

\section{Acoustic radiation force imaging}

Ultrasound imaging was performed in the same testing room where CMG was performed. A pediatric urology nurse placed a urodynamic catheter into the bladder and drained the bladder. A rectal catheter was not used. The bladder catheter was left in place for subsequent CMG. Ultrasound imaging then was performed by one of two pediatric radiologists (EAS, JRD). All studies were performed with the patient in a supine position. The bladder was imaged in a nearly empty state, with only a small amount of fluid separating the anterior and posterior walls of the bladder. If the volume of the fluid in the bladder was not sufficient, fluid was either removed via the catheter, or sterile saline was instilled into the bladder via the catheter by the pediatric urology nurse. The authors chose to study the bladder in the near-empty state, as it could be easily reproducible without the use of a catheter in the future if this technique became routine clinical practice. The thickness of the bladder wall is also greater when the bladder is nearly empty, allowing more reliable placement of ARFI regions-of-interest $(\mathrm{ROI})$ in the bladder wall.

Demographic data was recorded. All ultrasound imaging was performed using the same ultrasound system (Acuson 3000, Siemens Medical Solutions USA, Malvern, PA, U.S.) and the same 9L4 linear transducer. Measurement of the maximum thickness of the anterior wall of the bladder was recorded in millimeters. A total of nine elastography measurements of the bladder wall were obtained using 2D shear wave elastography (virtual touch tissue imaging quantification [VTIQ] method) (Fig. 1) and nine measurements were obtained using point shear wave elastography (virtual touch quantification [VTQ] method) (Fig. 2). For each method,

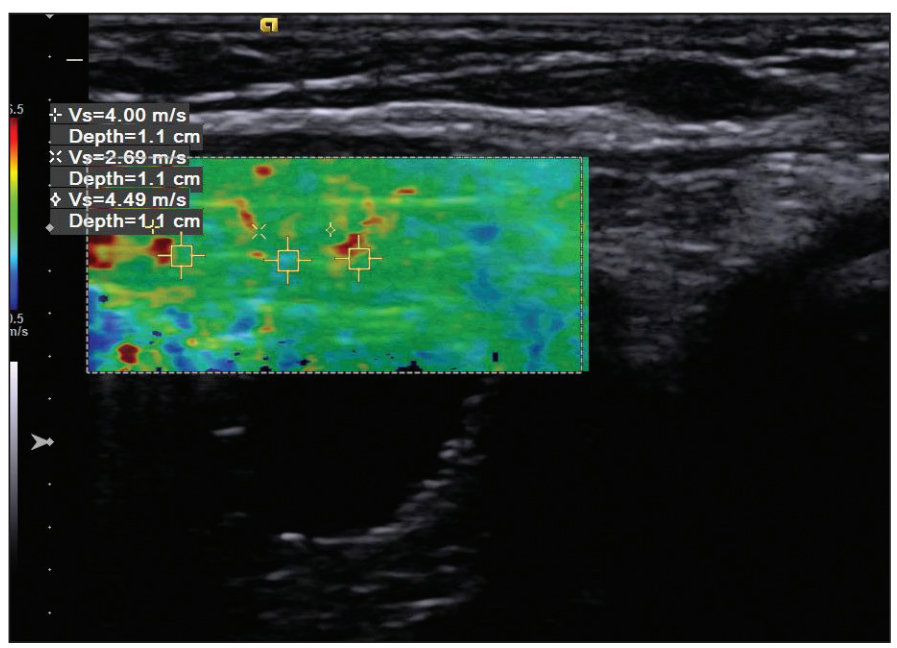

Fig. 1. Example of the method for $2 \mathrm{D}$ shear wave elastography (VTIO) measurement from the anterior bladder wall. $A$ total of three separate measurements were made from the same image for each area.

measurements were made in the following locations: three anterior wall, three left lateral wall, three right lateral wall. The ROI was placed manually by the radiologist to incorporate as much of the bladder wall as possible while avoiding adjacent tissues.

\section{Cystometrography testing and interpretation}

Following completion of the ultrasound, CMG was performed by the pediatric urology nurse using the existing bladder catheter. The patient remained in the supine position. Room temperature normal saline was infused from 10-20 $\mathrm{ml} /$ minute. All pressures were measured with a transducer and recorded by the pediatric urology nurse in

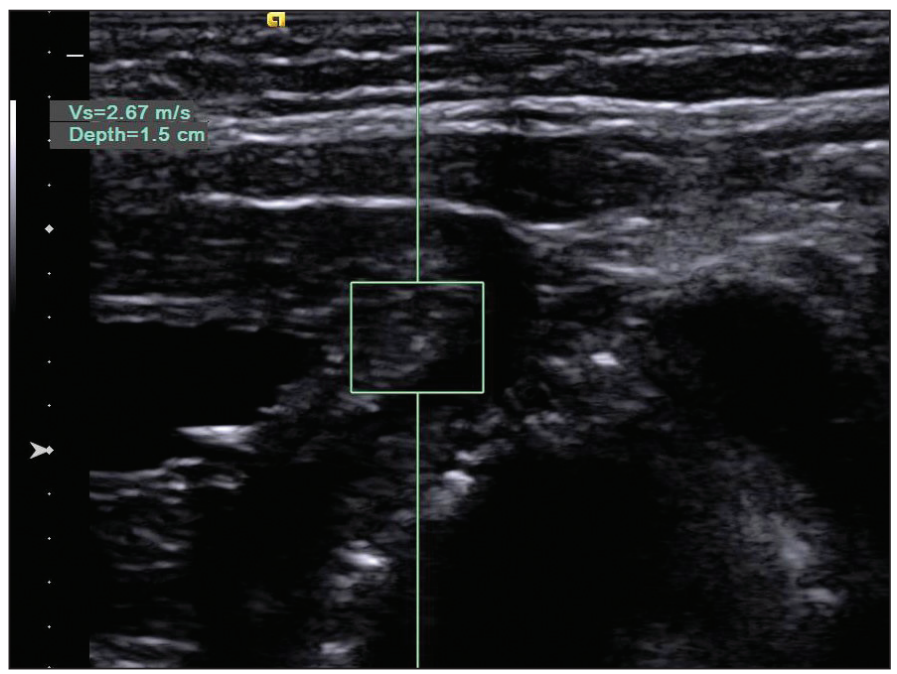

Fig. 2. Example of the method for point shear wave elastography (VTO) measurement from the left lateral bladder wall. For each area (anterior, right, left) three separate region of interest placements were performed. 
real time. CMG data was interpreted by a single pediatric urologist (KHK). The following parameters were recorded for all study subjects: detrusor leak point pressure or endfilling detrusor pressure; maximum detrusor pressure; actual bladder capacity; expected bladder capacity (EBC) ([age in years +2$] \times 30 \mathrm{~mL}$ if greater than one year of age and [7 $\mathrm{x}$ weight in $\mathrm{kg}$ ] if less than one year of age); bladder compliance (change in volume divided by the change in detrusor pressure in $\mathrm{ml} / \mathrm{cmH}_{2} \mathrm{O}$ ); voided volume; postvoid residual; and detrusor overactivity. All parameters were consistent with the International Children's Continence Society and the International Urogynecological Association/International Continence Society's defined terminology. ${ }^{9,10}$

To allow for a standardized comparison across ages, Wahl's dimensionless number was calculated for each patient to reflect bladder compliance $\left(100 \mathrm{~N}_{\text {Wahl }}{ }^{-1}=100\right.$ [volume at bladder capacity/volume at mean EBC]/[pressure at bladder capacity/pressure at mean EBC]), with a value of 33 or less representing "worrisome" or "unsafe" standardized compliance. ${ }^{11,12}$

\section{Statistical analysis}

Continuous data were summarized as means and standard deviations, while categorical data were summarized as counts and percentages. The relationship between bladder wall stiffness (shear wave speed in $\mathrm{m} / \mathrm{s}$ ) and CMG measurements was performed using Spearman correlation ( $r$ ) for nonparametric data. A p-value $<0.05$ was considered statistically significant. Statistics were calculated using MedCalc Statistical Software version 19.2 (MedCalc Software Ltd, Ostend, Belgium; https://www.medcalc.org).

\section{Results}

A total of 25 individual patients, including 15 boys and 10 girls, were enrolled, with a median age of two years (interquartile range [IQR] 0.75-6). Patient characteristics are summarized in Table 1. Shear wave speed obtained using 2D shear wave elastography technique was $2.37 \pm 0.51 \mathrm{~m} / \mathrm{s}$, and shear wave speed obtained using point shear wave elastography technique was $1.95 \pm 0.63 \mathrm{~m} / \mathrm{s}$. Mean decompressed bladder wall thickness was $3.9 \pm 1.8 \mathrm{~mm}$.

The most common clinical indication for CMG was spinal dysraphism (18 patients), among which 15 patients had a diagnosis of myelomeningocele. Adequate ultrasound elastography and CMG data were obtained in all patients. There were three $(12 \%)$ patients that did not have all nine ARFI measurements due to technical factors, most frequently patient motion.

CMG was considered abnormal in all but one patient. Abnormalities included worrisome or unsafe standardized bladder compliance in 16 (64\%), bladder capacity below EBC in $19(76 \%)$, and detrusor overactivity in $16(64 \%)$.
Only one patient was able to void spontaneously but had an elevated postvoid residual.

There was no statistically significant correlation between 2D shear wave speed measurements and bladder capacity $(r=-0.36, p=0.09)$, bladder compliance $(r=-0.35, p=0.1)$, leak volume $(r=0.16, p=0.58)$, or end-filling detrusor pressure $(r=0.36, p=0.21)$. Likewise, there was no statistically significant correlation between point shear wave speed measurements and bladder capacity $(\mathrm{r}=-0.08, \mathrm{p}=0.7)$, bladder compliance $(r=-0.22, p=0.31)$, leak volume $(r=-0.22, p=0.43)$, or end-filling detrusor pressure $(r=-0.46, p=0.1)$. There was no significant correlation between bladder wall thickness and bladder capacity $(\mathrm{r}=0.38, \mathrm{p}=0.06)$, bladder compliance $(r=0.4, p=0.05)$, leak volume $(r=0.28, p=0.3)$, or end-filling detrusor pressure $(r=0.1, p=0.72)$.

\section{Discussion}

Children with underlying diagnoses such as spina bifida, posterior urethral valves, spinal cord injury, and tumors of the central nervous system are particularly vulnerable to developing renal insufficiency if poor bladder compliance is not addressed appropriately. Testing requires insertion of catheters into the urethra and sometimes rectum, and therefore, urodynamic evaluation can be uncomfortable and poorly tolerated for pediatric patients, particularly those with intact sensation or unaccustomed to intermittent catheterization. Additionally, patients must remain still and calm during testing for the results to be readily interpretable. Identifying a non-invasive testing method, such as ultrasound ARFI, that could help identify abnormal bladder wall fibrosis and function and prevent subsequent upper urinary tract disease in the future would be ideal.

In the current study, we found no significant correlation between stiffness of the bladder wall, as measured by ultrasound shear wave elastography, and abnormal bladder capacity or compliance in children. These findings are of clinical relevance in that ARFI does not appear comparable to CMG in assessing bladder dynamics. Our results are distinct from those of previously published studies in which ultrasound elastography or vibrometry parameters correlate with bladder wall mechanical changes. ${ }^{8,13}$ Sturm et al similarly performed ultrasound elastography of the bladder wall in 23 pediatric patients. In that study, subjects were divided into two categories based on CMG parameters (normal, compliant bladders and abnormal, non-compliant bladders). They performed elastography measurements with the bladder at different levels of filling, ranging from empty to $100 \%$ of predicted bladder capacity to maximum bladder filling. The results showed significantly higher mean shear wave speed of the anterior wall in non-compliant compared to compliant bladders at all endpoints evaluated throughout bladder filling. The authors concluded that elastography may 


\begin{tabular}{|c|c|c|c|c|c|c|c|}
\hline Patient & Age (years) & Gender & Diagnosis & $\%$ EBC & $\begin{array}{c}\text { Wahl's dimensionless } \\
\text { number }\end{array}$ & $\begin{array}{l}\text { Detrusor } \\
\text { overactivity }\end{array}$ & $\begin{array}{c}\text { Anti- } \\
\text { cholinergics }\end{array}$ \\
\hline 1 & 6 & Male & Myelomeningocele & 126 & 125 & Yes & Yes \\
\hline 2 & 2 & Male & Myelomeningocele & 107 & 12 & No & Yes \\
\hline 3 & 13 & Male & Neurogenic bladder & 40 & 28 & No & Yes \\
\hline 4 & 3 & Female & Myelomeningocele & 29 & 2 & No & Yes \\
\hline 5 & 9 & Male & Myelomeningocele & 55 & 42 & No & Yes \\
\hline 6 & 5 & Male & Myelomeningocele & 55 & 10 & Yes & Yes \\
\hline 7 & 6 & Male & Enuresis & 12 & 63 & Yes & No \\
\hline 8 & 2 & Male & Neurogenic bladder & 87 & 52 & Yes & No \\
\hline 9 & 5 months & Female & Myelomeningocele & 42 & 10 & Yes & No \\
\hline 10 & 7 & Male & Myelomeningocele & 37 & 37 & Yes & Yes \\
\hline 11 & 5 & Female & Neurogenic bladder & 39 & 12 & Yes & Yes \\
\hline 12 & 2 & Female & Myelomeningocele & 23 & 23 & Yes & Yes \\
\hline 13 & 2 & Male & Myelomeningocele & 52 & 9 & Yes & No \\
\hline 14 & 4 & Female & Tethered cord & 158 & 24 & Yes & Yes \\
\hline 15 & 8 & Female & Myelomeningocele & 100 & 15 & No & Yes \\
\hline 16 & 3 months & Female & Myelomeningocele & 122 & 22 & Yes & No \\
\hline 17 & 15 & Female & Enuresis & 57 & 33 & No & No \\
\hline 18 & 8 & Male & Posterior urethral valves & 57 & 8 & Yes & No \\
\hline 19 & 9 months & Male & Myelomeningocele & 53 & 11 & Yes & No \\
\hline 20 & 2 & Male & Myelomeningocele & 68 & 40 & Yes & No \\
\hline 21 & 6 months & Female & Lipomeningocele & 59 & 69 & No & No \\
\hline 22 & 9 months & Male & Lipomyelomeningocele & 200 & 120 & No & No \\
\hline 23 & 2 months & Male & Posterior urethral valves & 43 & 26 & No & No \\
\hline 24 & 10 months & Female & Myelomeningocele & 65 & 29 & Yes & No \\
\hline 25 & 3 months & Female & Myelomeningocele & 79 & 54 & Yes & No \\
\hline
\end{tabular}

have value in distinguishing between normal and abnormal, non-compliant bladders. ${ }^{8}$

At first glance, the results found by Sturm and colleagues may seem contradictory to our results. On further examination, however, the results are complimentary, as Sturm and colleagues did not find any significant difference in elastography values between compliant and non-compliant bladders when the bladder was empty. This suggests that the elevated elastography values seen in the full bladder may not reflect a fixed tissue property, such as fibrosis or scarring, but instead must be due to some dynamic change in the bladder wall, possibly caused by different orientations of the bladder wall components as the tissue is maximally stretched. Changes in wall tension would, therefore, be expected over the course of bladder filling, given the elastic properties of the bladder wall tissue..$^{14-16}$ In support of this theory, it has been shown in other tissues that elevated elastography values can be seen with processes other than fibrosis, such as hepatic passive vascular congestion. ${ }^{17}$ Elevated elastography values reflect increased tissue stiffness, which can be a result of fibrosis but which occur in non-fibrotic and dynamic processes as well. ${ }^{18,19}$
Unlike other investigations, our study included patients less than five years of age, which could also explain discrepant results. We felt it important to include younger patients, as most subjects in this study had a diagnosis of myelomeningocele. Such patients are typically followed from birth for changes in urodynamics that might affect medical and surgical management. In infants or toddlers unaccustomed to catheterization, performing an invasive assessment can be traumatic for patient and family alike. The utility of ARFI in this age group, therefore, is of paramount importance. We recognize normal bladder compliance changes with age due to an increase in bladder capacity but not increased detrusor pressure..$^{20}$ We accounted for these physiological alterations over time by calculating Wahl's dimensionless number to normalize bladder capacity.

We acknowledge that our study has limitations. First, we only evaluated the bladder at minimal bladder capacity. This was done because the goal of the study was to determine if ultrasound elastography could non-invasively evaluate for bladder dysfunction to minimize the burden on patients and caregivers. It was felt that a minimally full bladder would be the most reproducible scenario in the daily clinical set- 
ting with the absence of catheter insertion. It would also allow for better evaluation of patients who otherwise are unable to fill to their EBC. Second, ARFI was performed by more than one radiologist, which could potentially introduce observer bias. Additionally, pathological review of bladder tissue was not included in our study design. Therefore, we have no histological evidence to support whether bladders of these subjects could be considered abnormal. Instead, we relied on the clinical gold standard of urodynamic testing to determine which patients had bladder dysfunction. Finally, our sample size remains relatively small, although our sample size was similar to that of prior investigations evaluating imaging in children with bladder dysfunction. A larger study could possibly reveal statistically significant associations between ultrasound-derived bladder wall stiffness and CMG findings.

\section{Conclusions}

Our study found no association between bladder wall stiffness, as measured by ultrasound, and CMG-derived bladder compliance or capacity in children with nearly empty bladders. Although other investigators have found significant correlation between elevated bladder wall stiffness by elastography and bladder dysfunction in maximally filled bladders, together these results imply that the elevated elastography values may not represent a fixed tissue property, such as fibrosis, and instead could relate to dynamic changes in bladder wall structure during filling and stretch.

Competing interests: The authors do not report any competing personal or financial interests related to this work.

This paper has been peer-reviewed.

\section{References}

1. McGuire EJ, Woodside JR, Borden TA, et al. Prognostic value of urodynamic testing in myelodysplastic patients. J Urol 1981;126:205-9. https://doi.org/10.1016/S0022-5347(17)54449-3

2. Ghoniem GM, Roach MB, Lewis VH, et al. The value of leak pressure and bladder compliance in the urodynamic evaluation of meningomyelocele patients. J Urol 1990;44:1440-2. https://doi.org/10.1016/ S0022-5347(17)39761-6
3. Bota S, Herkner H, Sporea I, et al. Meta-analysis: ARFI elastography versus transient elastography for the evaluation of liver fibrosis. Liver Int 2013;33:1138-47. https://doi.org/10.1111/liv.12240

4. Barr RG, Ferraioli G, Palmeri ML, et al. Elastography assessment of liver fibrosis: Society of Radiologists in Ultrasound consensus conference statement. Ultrasound Q 2016;32:94-107. https://doi.org/10.1097/ RUQ.0000000000000209

5. Dillman JR, Heider A, Bilhartz JL, et al. Ultrasound shear wave speed measurements correlate with liver fibrosis in children. Pediatr Radiol 2015;45:1480-8. https://doi.org/10.1007/s00247-015-3345-5

6. Landau EH, Jayanthi VR, Churchill BM, et al. Loss of elasticity in dysfunctional bladders: Urodynamic and histochemical correlation. J Urol 1994;152:702-5. hitps://doi.org/10.1016/S0022-5347(17)32685-X

7. Sturm RM, Cheng EY. The management of the pediatric neurogenic bladder. Curr Bladder Dysfunct Rep 2016;11:225-33. https://doi.org/10.1007/s1 1884-016-0371-6

8. Sturm RM, Yerkes EB, Nicholas JL, et al. Ultrasound shear wave elastography: A novel method to evaluate bladder pressure. J Urol 2017;198:422-9. https://doi.org/10.1016/i.juro.2017.03.127

9. Austin PF, Bauer SB, Bower W, et al. The standardization of terminology of lower urinary tract function in children and adolescents: Update report from the Standardization Committee of the International Children's Continence Society. J Urol 2014;191:1863-5. https://doi.org/10.1016/i.juro.2014.01.110

10. Haylen BT, de Ridder D, Freeman RM, et al. An International Urogynecological Association (IUGA)/ International Continence Society (ICS) joint report on the terminology for female pelvic floor dysfunction. Neurourol and Urodyn 2010;29:4-20. https://doi.org/10.1002/nau.20798

11. Wahl EF, Lerman SE, Lahdes-Vasama $\Pi$, et al. Measurement of bladder compliance can be standardized by a dimensionless number: theoretical perspective. BJU Int 2004;94:895-7. https://doi.org/10.1111/ j.1464-410X.2004.05054.x

12. Wahl EF, Lerman SE, Lahdes-Vasama TT, et al. Measurement of bladder compliance can be standardized by a dimensionless number: clinical perspective. BJU Int 2004;94:898-900. https://doi.org/10.1111/ j.1464-410X.2004.05055.x

13. Bayat $M$, Kumar $V$, Denis $M$, et al. Correlation of ultrasound bladder vibrometry assessment of bladder compliance with urodynamic study results. PLoS One 2017;12:e0179598. https://doi.org/10.1371/ journal.pone.0179598

14. Li C, Guan G, Zhang F, et al. Quantitative elasticity measurement of urinary bladder wall using laserinduced surface acoustic waves. Biomed Opt Express 2014;5:4313-28. https://doi.org/10.1364/ BOE.5.004313

15. Nenadic I, Mehrmohammadi M, Urban MW, et al. In vivo patient measurements of bladder elasticity using ultrasound bladder vibrometry (UBV). Conf Proc IEEE Eng Med Biol Soc 2013;2013:113-6. https://doi.org/10.1109/EMBC.2013.6609450

16. Nenadic IZ, Qiang B, Urban MW et al. Ultrasound bladder vibrometry method for measuring viscoelasticity of the bladder wall. Phys Med Biol 2013;58:2675-95. https://doi.org/10.1088/0031$9155 / 58 / 8 / 2675$

17. DiPaola FW, Schumacher KR, Goldberg CS, et al. Effect of Fontan operation on liver stiffness in children with single ventricle physiology. Eur Radiol 2017;27:2434-42. https://doi.org/10.1007/s00330016-4614-x

18. Brandenburg JE, Eby SF, Song P, et al. Ultrasound elastography: the new frontier in direct measurement of muscle stiffness. Arch Phys Med Rehabil 2014;95:2207-19. https://doi.org/10.1016/i. apmr.2014.07.007

19. Chernak LA, DeWall RJ, Lee KS et al. Length and activation dependent variations in muscle shear wave speed. Physiol Meas 2013;34:713-21. https://doi.org/10.1088/0967-3334/34/6/713

20. Neveus $T$, von Gontard $A$, Hoebeke $P$ et al. The standardization of terminology of lower urinary tract function in children and adolescents: Report from the Standardization Committee of the International Children's Continence Society. J Urol 2006;176:314-24. https://doi.org/10.1016/S0022-5347(06)00305-3

Correspondence: Dr. Kate H. Kraft, Division of Pediatric Urology, Department of Urology, University of Michigan Health System, Ann Arbor, MI, United States; kraftk@med.umich.edu 